\title{
Successful Treatment of Aluminum Phosphide Poisoning with Continuous Veno-venous Hemofiltration: A Case Report
}

\author{
Ayça Açıkalın ${ }^{1}$ Nezihat Rana Dişel ${ }^{1}$, Emre Karakoç², Selçuk Matyar ${ }^{3}$, Ahmet Sebe \\ 'Department of Emergency Medicine, Çukurova University School of Medicine, Adana, Turkey \\ ${ }^{2}$ Department of Intensive Care, Çukurova University School of Medicine, Adana, Turkey \\ ${ }^{3}$ Department of Medical Biochemistry, Çukurova University School of Medicine, Adana Turkey
}

\begin{abstract}
Introduction: We aim to discuss the case of a patient with aluminum phosphide (AP) poisoning in a suicide attempt and who was successfully treated with continuous veno-venous hemofiltration (CVVH).

Case Report: A-24-year old lady was admitted to a hospital with the complaints of dizziness, numbness in the face, and nausea and vomiting after taking two tablets of AP. She was transferred to our emergency clinic $10 \mathrm{~h}$ later because of persistent hypotension and metabolic acidosis. Her Glasgow Coma Scale score was 13 on admission. Her vital signs were as follows: blood pressure, 80/50 $\mathrm{mmHg}$; pulse rate, 128/min; pulse oximetry, 90\%; and respiration rate, 26/min. Her laboratory tests revealed the following: $\mathrm{pH}, 7.13$; bicarbonate level, $10.1 \mathrm{mmol} / \mathrm{L}$; and serum lactate level, $10.2 \mathrm{mmol} / \mathrm{L}$. Other blood test results were normal. CVVH (Prismaflex hemofiltration set M150) was started in the intensive care unit because hypotension and metabolic acidosis were persistent; oliguria was added despite fluid resuscitation and inotrope infusion. Hemofiltration was continued until the $32^{\text {nd }}$ hour when blood pressure and blood gas levels returned to normal. She was discharged healthy on the $4^{\text {th }}$ day of hospitalization.
\end{abstract}

Conclusion: We suggest the utilization of CVVH in the early stages of AP poisoning before the development of multiple organ failure because AP causes metabolic acidosis and hypotension resistant to medical treatment.

Keywords: Aluminum phosphide, continuous veno-venous hemofiltration, poisoning

Received: 11.11.2015 Accepted:30.12.2015 Available Online Date: 04.02.2016

\section{Introduction}

Aluminum phosphide (AP) is a pesticide that has been used since the 1940s (1). It is a metal phosphide compound ( $\mathrm{Na}_{3} \mathrm{P}, \mathrm{AIP}$ ) and is used as a rodenticide and insecticide. AP in an evaporable tablet form is used in wheat storage to reduce the number of mice attacks. This form causes fatalities, in suicidal exposure in particular. In countries such as Turkey, Iran, and India, public awareness of deadly exposure results in oral intake during suicide attempts (1). Gastric hydrochloric acid (HCl) causes the release of phosphine in the stomach after swallowing metal phosphide compounds. Phosphine induces cellular lipid peroxidation by means of the inhibition of mitochondrial cytochrome oxidase and oxygen utilization. This influence usually presents with hypotension, acidosis, and multiple organ failure (MOF) resistant to treatment. Mortality is extremely high and there is no identified antidote. Gastric irrigation with potassium and the replacement of intravenous magnesium have been suggested for treatment (1).

Herein we aim to discuss the case of a patient with AP poisoning in a suicide attempt and who was successfully treated with continuous veno-venous hemofiltration.

\section{Case Report}

A-24-year old lady was admitted to an urban hospital with complaints of dizziness, numbness in the face, and nausea and vomiting after taking two tablets of AP. Gastric lavage was performed, and she was given n-acetylcysteine, magnesium sulfate, 
Table 1. The vital signs and laboratory results of the patient

\begin{tabular}{lccc} 
& Results of the first hospital admission & Before hemofiltration & After hemofiltration \\
\hline Blood pressure $(\mathrm{mmHg})$ & $120 / 60$ & $80 / 50$ & $110 / 70$ \\
\hline Pulse rate (per minute) & 124 & 128 & 86 \\
\hline Blood pH & 7.18 & 7.13 & 7.358 \\
\hline HCO3- $(\mathrm{mmol} / \mathrm{L})$ & 11.1 & 10.1 & 22.7 \\
\hline Lactate $(\mathrm{mmol} / \mathrm{L})$ & 7.5 & 10.2 & 2 \\
\hline Hemoglobin $(\mathrm{g} / \mathrm{dL})$ & 12 & 10.4 & 9.2 \\
\hline Hematocrit $(\%)$ & 38.4 & 32 & 28.1 \\
\hline BUN $(\mathrm{mg} / \mathrm{dL})$ & 10 & 26.1 & 12 \\
\hline Cr $(\mathrm{mg} / \mathrm{dL})$ & 0.87 & 1.1 & 0.57 \\
\hline BUN: blood urea nitrogen, Cr: creatinine, HCO3: blood bicarbonate & &
\end{tabular}

sodium bicarbonate, crystalloids, and inotropes to control hypotension. She was transferred to our emergency clinic $10 \mathrm{~h}$ later because of hypotension resistant to medical treatment and persistent metabolic acidosis. She had confusion, and her Glasgow Coma Scale score was 13 on admission. Her vital signs were as follows: blood pressure, $80 / 50 \mathrm{mmHg}$; pulse rate, 128/minute; pulse oximetry, 90\%; and respiration rate, 26/min She had pallor; her skin was wet, and the capillary filling time was $>2 \mathrm{~s}$.

Her laboratory test results revealed the following: $\mathrm{pH}, 7.13$; bicarbonate level, $10.1 \mathrm{mmol} / \mathrm{L}$; and serum lactate level, $10.2 \mathrm{mmol} / \mathrm{L}$. Other biochemical and hematologic blood test results were within normal ranges. She had already received medical treatment for hypotension and metabolic acidosis and was still under inotrope infusion. Continuous veno-venous hemofiltration (Prismaflex hemofiltration set M150; Baxter, Lund, Sweden) was started in the intensive care unit. Magnesium sulfate at a dose of $4 \mathrm{~g} /$ day and inotrope infusion were simultaneously given. Hemofiltration was continued until the $32^{\text {nd }}$ hour when the blood pressure, blood gas levels, and tissue perfusion signs returned to normal. She had no abnormality except anemia after hemofiltration. The vital signs and laboratory results of the patient are summarized in Table 1. She was discharged healthy without any sequela on the $4^{\text {th }}$ day of hospitalization. Written consent of the patient was obtained for preparing this case report.

\section{Discussion}

Metal phosphide compounds are widely used as rodenticides and insecticides in Turkey (2). AP is manufactured in the solid form and is stored as packed huge tablets and granules. These tablets turn into the gaseous form in 1 or $2 \mathrm{~h}$ following the destruction of the protective package. Poisoning occurs when this gas is inhaled or when tablets are accidentally or intentionally swallowed in a suicide attempt (1).

Ingested metal phosphide compounds form phosphine in the stomach following a chemical reaction with $\mathrm{HCl}$. Exposure to phosphine at a dose of $1400 \mathrm{mg} / \mathrm{m}^{3}$ in $30 \mathrm{~min}$ can be fatal (WHO, 1988). There are many reports on cases from countries such as India where there are accidentally poisoning cases due to the careless usage of
AP in the storage of agricultural products (1). Headache, dyspnea, chest discomfort, and numbness are the frequently seen complaints in these case reports. It was reported in a retrospective study that the complaints of patients who were poisoned after pesticide application because of a lack of protective measures were headache in $31.8 \%$ of the patients, chest discomfort in $27.3 \%$, and dyspnea in $31.8 \%(1,3)$. Our patient was admitted to the emergency clinic with sensory motor complaints such as headache, dizziness, and numbness in the face.

Intentional oral intake in a suicide attempt is a frequently seen form of exposure of AP, in Iran and India in particular (1). When intentionally ingested, phosphine formed from AP causes hypotension resistant to medical treatment, persistent metabolic acidosis, and MOF by lipid peroxidation due to the inhibition of mitochondrial cytochrome oxidase enzyme and oxygen utilization in cellular basis. There is no antidote of AP, and the mortality rate because of this poisoning is extremely high $(1,4)$.

The mortality rate of AP poisoning was reported to be as high as $65 \%$ in a retrospective study by Singh et al. (5) The study by Moghadamnia et al. (6) in Iran revealed 19 mortalities from 21 AP poisoning patients. The reported mortality rate of AP poisoning in literature is $37-100 \%(1,6)$. This poisoning, which has a high mortality rate, has no specific treatment options. There are reports of particular effective treatment modalities that may reduce mortality rates. Gastric lavage with potassium permanganate is one of these treatment options suggested in early admissions. However, the inhalation and diffusion of phosphine make this decontamination option ineffective for decreasing absorption.

Symptomatic treatment modalities are major management approaches. Death is inevitable in patients with resistant hypotension, metabolic acidosis, and poor tissue perfusion. In this phase, inotropes for resistant hypotension and sodium bicarbonate for metabolic acidosis have been reported in many studies as supportive measures (1). Although it is not an antidote, magnesium is suggested in the treatment of such patients. Magnesium is a cofactor in the synthesis of glutathione and other antioxidants. Hypomagnesemia 
is seen after AP poisoning (1). In a study with 50 patients, the mortality rate of patients who received magnesium was $20 \%$, while it was $40 \%$ who did not (7).

Our patient had taken two AP tablets in a suicide attempt. She was started on dopamine, magnesium, and sodium bicarbonate infusions. She was admitted to the intensive care unit to perform continuous veno-venous hemofiltration to treat resistant hypotension and metabolic acidosis. This is a method to treat acid-base and electrolyte disorders of hemodynamically unstable patients. In poisoned patients, ongoing metabolic acidosis and hypotension resistant to medical treatment lead to myocardial depression and MOF. There are several reports on the successful utilization of hemofiltration in poisoning cases with hypotension and metabolic acidosis, especially in patients with salicylate and metformin overdoses (8). There is a case report of a patient who underwent continuous veno-venous hemofiltration for AP poisoning. Unfortunately, this patient died because of ventricular tachycardia unresponsive to cardioversion (9). Nasa et al. (10) reported two patients with acute AP poisoning who survived after continuous renal replacement therapy. We started hemofiltration before MOF occurred in our patient. Long periods of metabolic acidosis and hypotension cause a high mortality rate due to irreversible cardiac problems and additive MOF. We started hemofiltration as soon as resistant hypotension and metabolic acidosis were diagnosed and the serum creatinine level was $1.1 \mathrm{mg} / \mathrm{dL}$. The patient was under continuous veno-venous hemofiltration for 32 h. No laboratory abnormality and complication were defected. She was discharged healthy on the 4th day of hospitalization.

\section{Conclusion}

We suggest hemofiltration in the early stages of clinical follow-up as a treatment choice in poisoning patients, particularly if the agent causes metabolic acidosis and resistant hypotension. In poisoning with AP, hemofiltration may be a life-saving method as it prevents progression to cardiovascular complications and MOF due to prolonged metabolic acidosis. Furthers studies are needed to determine the effectiveness of hemofiltration in AP poisoning.

Informed Consent: Written informed consent was obtained from patient who participated in this case.
Peer-review: Externally peer-reviewed.

Author Contributions: Concept - A.A., E.K.; Design - A.A., N.R.D., E.K.; Supervision - A.A., N.R.D.; Materials - A.A., E.K., A.S., S.M.; Data Collection and/or Processing - A.A., N.R.D., A.S., E.K., S.M.; Analysis and/or Interpretation - A.A., N.R.D., S.M., A.S., E.K.; Literature Review - A.A.; Writer - A.A., N.R.D., A.S.; Critical Review - E.K., S.M., A.S.

Conflict of Interest: The authors declared no conflict of interest.

Financial Disclosure: The authors declared that this study has received no financial support.

\section{References}

1. Moghadamnia AA. An update on toxicology of aluminum phospide. DARU Journal of Pharmaceutical Sciences, 2012; 20: 25. [CrossRef]

2. Satar S, Seydaoglu G, Akpinar A, Sebe A, Karakoc E, Gumusay U et al. Trends in acute adult poisoning in a ten-year period in Turkey: factors affecting the hazardous outcome. Bratisl Lek Listy 2009; 110: 404-11.

3. Katira R, Elhence GP, Mehrotra ML, Srivastava SS, Mitra A, Agarwala R, et al. A study of aluminum phosphide (AIP) poisoning with special reference to electrocardiographic changes. J Assoc Physicians India 1990; 38: 471-3.

4. Mehrpour O, Jafarzadeh M, Abdollahi M. A systematic review of aluminium phosphide poisoning. Arh Hig Rada Toksikol, 2012; 63: 61-73. [CrossRef]

5. Singh D, Jit I, Tyagi S. Changing trends in acute poisoning in Chandigarh zone: a 25-year autopsy experience from a tertiary care hospital in northern India. Am J Forensic Med Pathol, 1999; 20: 203-10. [CrossRef]

6. Moghadamnia AA, Firoozjahi AR, Javadian SH, Dibavand N. Aluminium phosphide poisoning in mice and the procedure for its management. $J$ Babol Univ Med Sci 2000; 2: 25-33.

7. Chugh SN, Kolley T, Kakkar R, Chugh K, Sharma A. A critical evaluation of anti-peroxidant effect of intravenous magnesium in acute aluminium phosphide poisoning. Magnes Res 1997; 10: 225-30.

8. Jadhav AP, Nusair MB, Ingole A, Alpert MA. Unresponsive ventricular tachycardia associated with aluminum phosphide poisoning. Am J Emerg Med 2012; 30: 633.e3-5. [CrossRef]

9. Finkle SN. Should dialysis be offered in all cases of metformin-associated lactic acidosis? Crit Care 2009; 13: 110. [CrossRef]

10. Nasa P, Gupta A, Mangal K, Nagrani SK, Raina S, Yadav R. Use of continuous renal replacement therapy in acute aluminum phosphide poisoning: a novel therapy. Ren Fail 2013; 35: 1170-2. [CrossRef] 